\title{
Duality in a fermionlike formulation for the electromagnetic field
}

\author{
Everton M. C. Abreu* and Marcelo Hott ${ }^{\dagger}$ \\ Departamento de Física e Química, Universidade Estadual Paulista, Av. Ariberto Pereira da Cunha 333, Guaratinguetá, 12500-000, \\ São Paulo, SP, Brazil
}

(Received 14 February 2000; published 27 June 2000)

\begin{abstract}
We employ the Dirac-like equation for the gauge field proposed by Majorana to obtain an action that is symmetric under duality transformation. We also use the equivalence between duality and chiral symmetry in this fermionlike formulation to show how the Maxwell action can be seen as a mass term.

PACS number(s): 11.15.-q, 11.10.Ef, 11.30.Cp, 11.30.Rd
\end{abstract}

\section{INTRODUCTION}

Recently, many papers have been published exploring the issue of duality. This production has been motivated by the relation between duality symmetry and theories with strong and weak coupling [1]. Another motivation has been due to the presence of various duality symmetries in string theories [2], specifically, the target space duality ( $T$ duality), which is the symmetry of the low energy effective field theory, and $S$ duality (the generalization of the electric or magnetic duality) which is the invariance under the $S L(2, R)$ duality transformations of the equations of motion for the bosonic sector of the heterotic string. In addition to these, a very important interest resides in the electrically and magnetically charged black holes in the semiclassical view [3].

Consequently, one of the first dualities observed, the electric-magnetic duality in Maxwell's equations, has received great attention. However, the problem that arises in preserving the duality symmetry and, at the same time, the manifest Lorentz covariance when the duality is implemented is a very difficult obstacle. The main objective in the literature is to construct a duality symmetric action which is also manifestly Lorentz invariant.

Schwarz and Sen (SS) [4] proposed a dual invariant action in which one more potential has been introduced generalizing the $T$ duality symmetric string action [5] to the case of the heterotic string. Although this formulation is not manifestly covariant it is classically and quantically Poincare invariant. One way to recover the manifest Lorentz invariance is to produce a nonpolynomial action, but this makes the quantization difficult. An alternative procedure is to use the Hamiltonian formalism in which, after the introduction of an infinite set of fields (this idea was first used to analyze chiral bosons $[6,7]$ ) it is possible to get suitable duality conditions [8]. The corresponding action, in ten dimensions, containing an infinite number of fields, is manifestly invariant under electromagnetic duality transformation. After compactification to four dimensions it results into a local Maxwell action with electric and magnetic sources [9]. The study of the dimensional dependence of the electromagnetic duality was carried out in [10] and the connection between duality and bosonization was shown in [11].

\footnotetext{
*Email address: everton@feg.unesp.br

${ }^{\dagger}$ Email addresss: hott@feg.unesp.br
}

In the approach of the source-free Maxwell theory in an arbitrary background geometry, the duality symmetry under general electric-magnetic field rotations can be implemented in a nonlocal way, as was introduced by Deser and Teitelboim (DT) [12]. If the manifest covariance is lost, we can construct quadratic actions for these models. It can be proved that this formulation is equivalent to the one of Schwarz-Sen [13] via path integral formalism. In Ref. [14] sources were introduced into these nonmanifestly invariant actions and the covariantization of this procedure has been accomplished in [15].

Khoudeir and Pantoja (KP) [16], getting back to the nonpolynomial action scheme, suggested a Lorentz invariant version of the SS model by using an auxiliary timelike constant vector in the action. This vector in fact violates the manifest covariance. Pasti, Sorokin, and Tonin (PST) [17], making good use of this idea, proposed a generalization of the DT, SS, and KP duality symmetric actions presenting the KP unit norm auxiliary vector as a Lorentz frame vector field that can be related to the gravitation interaction. The action constructed is manifestly covariant.

In this work we use the fermionlike formulation of Majorana $[18,19]$ for the Maxwell theory to propose an action that is invariant under relativistic and duality transformations. Fermionlike formulation for the electromagnetic theory has been studied extensively by Dvoeglazov [20], but connections with duality has not been explored. This paper is organized as follows: in Sec. II we review the work of Majorana and obtain a Dirac-like expression for the Maxwell equations in the absence of sources. This femionlike formulation encouraged us to explore the chiral aspects in the theory. This is accomplished in Sec. III where we show that duality is a kind of chirality and in this context we propose our action which is invariant under duality. In Sec. IV we demonstrate that the Poincare generators obeys the algebra on-shell. Finally, the conclusion and final observations are in Sec. V.

\section{FERMIONLIKE FORMULATION}

The well known Maxwell's equations are

$$
\nabla \cdot \mathbf{E}=\rho,
$$

$\nabla \cdot \mathbf{B}=0$, 


$$
\begin{aligned}
& \nabla \times \mathbf{B}-\frac{\partial \mathbf{E}}{\partial t}=\mathbf{j}, \\
& \nabla \times \mathbf{E}+\frac{\partial \mathbf{B}}{\partial t}=0 .
\end{aligned}
$$

Now we want to look for a fermionlike formulation of these equations based on the work of Majorana [19]. In his work Majorana enhanced the role in electrodynamics of the complex quantity $\mathbf{F}=\mathbf{E}-i \mathbf{B}$, which was emphasized lately by Weinberg [21] and others [22]. The main motivation for this construction is the well known fact that at the statistical level, the electric and magnetic fields $\mathbf{E}$ and $\mathbf{B}$ are connected (through the quantity $\mathbf{E}^{2}+\mathbf{B}^{2}$ ) to the local mean number of photons. Hence, an expression for the probability quantum wave of a photon can be given in terms of $\mathbf{E}$ and $\mathbf{B}$. It provides a meaning different from the usual one, where the electromagnetic four-potential is introduced.

The first step is to build elements and vectors that allow us to couple two equations into only one. To accomplish this we note that Eqs. (3) and (4), for $\mathbf{j}=0$, can be rewritten as

$$
i \frac{\partial \mathbb{E}}{\partial t}=\frac{1}{i}(\mathbf{s} \cdot \nabla) i \mathbb{B}
$$

and

$$
i \frac{\partial(i \mathrm{~B})}{\partial t}=\frac{1}{i}(\mathbf{s} \cdot \nabla) \mathbb{E}
$$

where

$$
\mathbb{E}=\left(\begin{array}{c}
E_{1} \\
E_{2} \\
E_{3}
\end{array}\right) \quad \text { and } \mathbb{B}=\left(\begin{array}{c}
B_{1} \\
B_{2} \\
B_{3}
\end{array}\right) \text {. }
$$

Note that we have defined $\mathbf{s}$ as three matrices $3 \times 3$, by $\left(s^{i}\right)_{j k}=-i \epsilon_{i j k}$, where $\epsilon_{i j k}$ is the Levi-Civita totally antisymmetric tensor normalized so that $\epsilon_{123}=1$ and $(\mathbf{s} \cdot \nabla) \mathbb{B}$ $=\left(s_{i}\right)^{j k} \partial_{j} B_{k}$.

With this definition the $s^{i}$ matrices have the explicit structure

$$
\begin{aligned}
s^{1} & =\left(\begin{array}{ccc}
0 & 0 & 0 \\
0 & 0 & -i \\
0 & i & 0
\end{array}\right), \quad s^{2}=\left(\begin{array}{ccc}
0 & 0 & i \\
0 & 0 & 0 \\
-i & 0 & 0
\end{array}\right), \\
s^{3} & =\left(\begin{array}{ccc}
0 & -i & 0 \\
i & 0 & 0 \\
0 & 0 & 0
\end{array}\right),
\end{aligned}
$$

which satisfy the angular-momentum algebra

$$
\left[s_{i}, s_{j}\right]_{-}=-i \epsilon_{i j k} s_{k} \quad(i, j, k=1,2,3),
$$

and in this way Eq. (5), for example, can be rewritten as

$$
i \frac{\partial E_{i}}{\partial t}=\left(s_{i}\right)^{j k} \cdot \partial_{j} B_{k}
$$

In order to express Eqs. (5) and (6) in a fermionlike formulation we define two important $6 \times 6$ matrices,

$$
\Gamma_{0}=\left(\begin{array}{cc}
\mathbb{I} & 0 \\
0 & -\mathbb{I}
\end{array}\right) \quad \text { and } \vec{\Gamma}=\left(\begin{array}{cc}
0 & \mathbf{s} \\
-\mathbf{s} & 0
\end{array}\right) \text {. }
$$

$\Gamma_{0}$ and $\vec{\Gamma}$ play the role of the Dirac gamma matrices, although they do not obey the usual gamma anticommutation relations. We can also introduce a matrix that is equivalent to Dirac's $\gamma_{5}$,

$$
\Gamma_{5}=\left(\begin{array}{ll}
0 & \mathbb{I} \\
\mathbb{I} & 0
\end{array}\right)
$$

where $I$ is the $3 \times 3$ identity matrix. One can check that $\left\{\Gamma_{5}, \Gamma_{\mu}\right\}=0, \mu=0, \ldots, 3$.

With these definitions Eqs. (5) and (6) can be put in a more compact form

$$
i \frac{\partial \Psi}{\partial t}=\frac{1}{i}(\mathrm{~S} \cdot \nabla) \Psi \Rightarrow\left(\partial_{t}+\mathrm{S} \cdot \nabla\right) \Psi=0,
$$

where $\mathrm{S}$ is

$$
S=\left(\begin{array}{ll}
0 & \mathbf{S} \\
\mathbf{S} & 0
\end{array}\right) .
$$

Equation (13) resembles the massless Dirac equation, and one can consider $\Psi$ as a (quantum) wave function for the photon of the type

$$
\Psi=\left(\begin{array}{c}
\mathbb{E} \\
i \mathbb{B}
\end{array}\right)
$$

and

$$
\bar{\Psi}=\left(\mathbb{E}^{\dagger} \quad i \mathbb{B}^{\dagger}\right)
$$

where $\bar{\Psi}=\Psi^{\dagger} \Gamma_{0}$ is an analogue of the Hermitian conjugated definition. Notice that only $\mathbf{E}$ and $\mathbf{B}$ have physical meaning and we will use $\mathbf{E}^{\dagger}$ and $\mathbf{B}^{\dagger}$ as auxiliary fields. So, we can note that $\Psi^{\dagger} \Psi=\mathbf{E}^{2}+\mathbf{B}^{2}$, with $\mathbf{E}^{\dagger}=\mathbf{E}$ and $\mathbf{B}^{\dagger}=\mathbf{B}$. Now we can see the importance of the complex notation in the construction of $\Psi$ which mimics a Dirac spinor.

In terms of the $\Gamma$ matrices we may rewrite Eq. (13) as

$$
\begin{aligned}
i \Gamma_{0} \frac{\partial \Psi}{\partial t} & =\frac{1}{i}\left(\Gamma_{0} \mathrm{~S} \cdot \nabla\right) \Psi \\
& =\frac{1}{i}(\vec{\Gamma} \cdot \nabla) \Psi \\
& \Rightarrow\left(\Gamma_{0} \partial_{t}+\vec{\Gamma} \cdot \nabla\right) \Psi=0
\end{aligned}
$$

or compactly 


$$
\Gamma^{\mu} \partial_{\mu} \Psi=0
$$

though it is not manifestly covariant.

\section{CHIRALITY AND DUALITY TRANSFORMATIONS}

We can also note that Eq. (19) is invariant under the chiral transformation

$$
\Psi \rightarrow \Psi=e^{i \theta \Gamma_{5}} \Psi
$$

In terms of the fields $\mathbb{E}$ and $\mathbb{B}$ we have

$$
\begin{aligned}
& \mathbb{E}=\cos \theta \mathbb{E}-\sin \theta \mathbb{B}, \\
& \mathbb{B}=\cos \theta \mathbb{B}+\sin \theta \mathbb{E},
\end{aligned}
$$

which is a rotation and, particularly for $\theta=-\pi / 2$, we recover the duality transformation.

This invariance can also be verified in the following Lagrangian density

$$
\mathcal{L}=i \alpha \bar{\Psi}\left(\Gamma_{0} \partial_{t}+\vec{\Gamma} \cdot \nabla\right) \Psi
$$

or compactly $\mathcal{L}=i \alpha \Psi \Gamma^{\mu} \partial_{\mu} \Psi$, where the vectors $\Psi$ and $\Psi^{\dagger}$ must be taken conveniently as independent. In order to provide the correct dimension of the action in the natural units $(\hbar=c=1)$ we have introduced a parameter $\alpha$ whose dimension is mass $^{-1}$ and does not modify the equations of motion.

In terms of $\mathbb{E}$ and $B$ Eq. (22) is given by

$$
\mathcal{L}=\alpha\left(i \mathbb{E}^{\dagger} \mathbb{E}+i \mathbb{B}^{\dagger} \dot{B}-\mathbb{E}^{\dagger} \mathbf{s} \cdot \nabla \mathbb{B}+\mathbb{B}^{\dagger} \mathbf{s} \cdot \nabla \mathbb{E}\right),
$$

which is first order in the time derivative and consequently has a Hamiltonian formulation. The physical quantities of the theory are real and the main role of the auxiliary fields is in the mathematical construction of the Maxwell equations as we will see below.

The equations of motion for $\mathbf{E}^{\dagger}$ and $\mathbf{B}^{\dagger}$ can be obtained from the Euler-Lagrange equations

$$
\frac{\partial \mathcal{L}}{\partial E_{i}}-\partial_{t} \frac{\partial \mathcal{L}}{\partial \dot{E}_{i}}-\partial_{j} \frac{\partial \mathcal{L}}{\partial\left(\partial_{j} E_{i}\right)}=0
$$

and

$$
\frac{\partial \mathcal{L}}{\partial B_{i}}-\partial_{t} \frac{\partial \mathcal{L}}{\partial \dot{B}_{i}}-\partial_{j} \frac{\partial \mathcal{L}}{\partial\left(\partial_{j} B_{i}\right)}=0
$$

with the following results,

$$
-i \frac{\partial \mathbb{E}^{\dagger}}{\partial t}=\frac{1}{i}(\mathbf{s} \cdot \nabla) i \mathbb{B}^{\dagger}
$$

and

$$
-i \frac{\partial\left(i \mathbb{B}^{\dagger}\right)}{\partial t}=\frac{1}{i}(\mathbf{s} \cdot \nabla) \mathbb{E}^{\dagger},
$$

which are the Hermitian conjugated of Eqs. (5) and (6), respectively. The equations of motion for $\mathbf{E}$ and $\mathbf{B}$ are obtained from

$$
\frac{\partial \mathcal{L}}{\partial E_{i}^{\dagger}}=0 \quad \text { and } \frac{\partial \mathcal{L}}{\partial B_{i}^{\dagger}}=0,
$$

and as a result we have Eqs. (5) and (6).

Equations (1) and (2) for $\rho=0$ comes from the fact that $\mathbf{E}$ and $\mathbf{B}$ are orthogonal to the propagation vector, namely

$$
\mathbf{k} \cdot \mathbf{E}=0 \text { and } \mathbf{k} \cdot \mathbf{B}=0 \text {. }
$$

The mass term: It is well known that a term like $\Psi \Psi$ breaks the chiral invariance. In this formulation it is given by

$$
\Psi \Psi=\mathbb{E}^{\dagger} \cdot \mathbb{E}-\mathbb{B}^{\dagger} \cdot \mathbb{B}
$$

which resembles the Maxwell Lagrangian density (with $\mathbf{E}^{\dagger}$ $=\mathbf{E}, \quad \mathbf{B}^{\dagger}=\mathbf{B}$ ) and highlights the connection between chiral symmetry and duality. A mass term in the Dirac form is not invariant under chiral transformation and the Maxwell Lagrangian is not invariant under duality transformation.

\section{THE GENERATORS OF THE POINCARÉ ALGEBRA}

Notice that, as the Schwarz-Sen model, Eq. (23) is not a Lorentz scalar. However, it is our task now to demonstrate that the theory is relativistically invariant as it obeys the Poincaré algebra.

We have computed the generators of the Poincaré algebra in the usual way, that is, by using the energy-momentum tensor as

$$
\Theta_{\mu \nu}=\frac{\partial \mathcal{L}}{\partial\left(\partial^{\mu} \phi_{i}\right)} \frac{\partial \phi_{i}}{\partial x^{\nu}}-g_{\mu \nu} \mathcal{L}
$$

where $\phi_{i}$ are the basic fields $E_{i}\left(E_{i}^{\dagger}\right)$ and $B_{i}\left(B_{i}^{\dagger}\right)$. Consequently the linear and angular momenta are given by

$$
\begin{aligned}
& H=P_{0}=\int d^{3} x \Theta_{00}=\alpha \int d^{3} x\left[\mathbb{E}^{\dagger} \mathbf{s} \cdot \nabla \mathbb{B}-\mathbb{B}^{\dagger} \mathbf{s} \cdot \nabla \mathbb{E}\right], \\
& P_{k}=\int d^{3} x \Theta_{0 k}=i \alpha \int d^{3} x\left[\mathbb{E}^{\dagger} \partial_{k} \mathbb{E}+\mathbb{B}^{\dagger} \partial_{k} \mathbb{B}\right],
\end{aligned}
$$

and

$M_{\mu \nu}=i \alpha \int d^{3} x\left[x_{\mu}\left(\mathbb{E}^{\dagger} \partial_{\nu} \mathbb{E}+\mathbb{B}^{\dagger} \partial_{\nu} \mathbb{B}\right)-x_{\nu}\left(\mathbb{E}^{\dagger} \partial_{\mu} \mathbb{E}+\mathbb{B}^{\dagger} \partial_{\mu} \mathbb{B}\right)\right]$,

where $\mu, \nu=0, \ldots, 3$ and $k=1, \ldots, 3$. Finally it can be shown in a straightforward calculation that they obey the Poincaré algebra on-shell, i.e.,

$$
\begin{array}{r}
\left\{P_{k}, P_{0}\right\}=0, \\
\left\{M_{\mu \nu}, P_{0}\right\}=0,
\end{array}
$$




$$
\begin{aligned}
\left\{M_{\mu \nu}, P_{k}\right\}= & g_{k \nu} P_{\mu}-g_{k \mu} P_{\nu}, \\
\left\{M_{\mu 0}, P_{k}\right\}= & g_{k \mu} P_{0}, \\
\left\{M_{\mu \nu}, M_{\lambda \sigma}\right\}= & g_{\nu \lambda} M_{\mu \sigma}-g_{\mu \sigma} M_{\nu \lambda} \\
& -g_{\nu \sigma} M_{\mu \lambda}-g_{\mu \lambda} M_{\nu \sigma} .
\end{aligned}
$$

So, we have demonstrated that the action in Eq. (23) describes a theory that is relativistically invariant on-shell.

\section{CONCLUSIONS}

We have analyzed an already known fermionlike formulation for the electromagnetic theory and proposed a corresponding Lagrangian invariant under duality transformation. This invariance can be seen as a particular case of chiral transformation in the fermionlike formulation. We have carried out our analysis in order to verify the Poincaré invariance of this formulation, and have found that, although not being manifestly covariant, it is relativistically invariant onshell. We also have compared the chiral variant mass term with the Maxwell Lagrangian, which is duality noninvariant. As a perspective for the future we intend to study the duality symmetry in other formulations of the electromagnetism.

\section{ACKNOWLEDGMENTS}

The authors would like to thank the Fundação de Amparo à Pesquisa do Estado de São Paulo (FAPESP), a Brazilian research agency, for financial support, Professors A. S. Dutra and C. Wotzasek for valuable discussions, and Professor V. Dvoeglazov for helpful comments.
[1] C. Montonen and D. Olive, Phys. Lett. 72B, 117 (1977); P. Goddard, J. Nyuts, and D. Olive, Nucl. Phys. B125, 1 (1977).

[2] A. Font, L. Ibañez, D. Lust, and F. Quevedo, Phys. Lett. B 249, 35 (1990); S. J. Rey, Phys. Rev. D 43, 526 (1991).

[3] S. Deser, M. Henneaux, and C. Teitelboim, Phys. Rev. D 55, 826 (1997)

[4] J. H. Schwarz and A. Sen, Nucl. Phys. B411, 35 (1994).

[5] A. Tseytlin, Phys. Lett. B 242, 163 (1990); Nucl. Phys. B350, 395 (1991).

[6] C. Wotzasek, Phys. Rev. Lett. 66, 129 (1991).

[7] B. McClain, Y. S. Wu, and F. Yu, Nucl. Phys. B343, 689 (1990).

[8] I. Bengtsson and A. Kleppe, Int. J. Mod. Phys. A 12, 3397 (1997).

[9] N. Berkovits, Phys. Lett. B 395, 28 (1997).

[10] C. Wotzasek, Phys. Rev. D 58, 125026 (1998).

[11] C. Wotzasek and R. Banerjee, Nucl. Phys. B527, 402 (1998).

[12] S. Deser and C. Teitelboim, Phys. Rev. D 13, 1592 (1976).

[13] H. O. Girotti, M. Gomes, V. O. Rivelles, and A. J. da Silva, Phys. Rev. D 56, 6615 (1997).

[14] S. Deser, A. Gomberoff, M. Henneaux, and C. Teitelboim,
Phys. Lett. B 400, 80 (1997).

[15] R. Medina and N. Berkovits, Phys. Rev. D 56, 6388 (1997).

[16] A. Khoudeir and N. Pantoja, Phys. Rev. D 53, 5974 (1996).

[17] P. Pasti, D. Sorokin, and M. Tonin, Phys. Lett. B 352, 59 (1995); Phys. Rev. D 52, R4277 (1995); 56, 2473 (1997).

[18] E. Majorana, "Scientific Manuscripts," deposited at the "Domus Galilaeana,' Pisa, quaderno 2, p. 101/1; 3, pp. 11, 160; 15, p. 16; 17, p. 83, 159; E. Majorana, Nuovo Cimento 9, 335 (1932).

[19] R. Mignani, E. Recami, and M. Baldo, Lett. Nuovo Cimento Soc. Ital. Fis. 11, 568 (1974);

[20] V. V. Dvoeglazov, Hadronic J. 16, 459 (1993); Apeiron 5, 69 (1998).

[21] S. Weinberg, in Lectures on Particles and Field Theory, edited by S. Deser and K. W. Ford (Englewood Clifts, NJ, 1965), p. 405.

[22] L. Landau and E. Lifshitz, Theorie du Champ (Mir, Moscow, 1966); D. Weingarten, Ann. Phys. (N.Y.) 76, 510 (1973); J. M. Leinaas, Nuovo Cimento A 15, 740 (1973); E. Recami and R. Mignami, Riv. Nuovo Cimento 4, 209 (1974). 